\title{
American Society for Horticultural Science
}

113 South West Street, Suite 200, Alexandria, Virginia 22314-2851, USA

Phone 703/836/4606 Fax 703/836/2024

July 2007

Dear ASHS-07 Conference Participants,

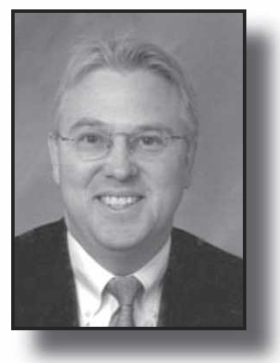

Welcome to the 104th annual conference of the American Society for Horticultural Science, which will be held from 16-19 July at the beautiful Four-Diamond Westin Kierland Resort and Spa in Scottsdale, Arizona. This conference facility promises to be one of the most spectacular sites that ASHS members have ever experienced. I can't wait! I look forward to this meeting every year. The conference provides an outstanding opportunity to share science and education, to network with colleagues, and to think about the future of horticultural science.

Each ASHS Annual Conference is unique, and this year is no exception.

This Program and Abstracts issue of HortScience contains summaries of technical reports, a list of participants, and a schedule of activities and events that will make this conference truly special. More than 690 abstracts were submitted for Poster and Oral Sessions for this year's conference. In addition, there are 29 Workshops, 3 Symposia, 4 Colloquia, and 2 Special Sessions covering the diversity that is Horticultural Science. The heart and soul of the American Society for Horticultural Science is the working groups. Members come together in these common-interest groups to share their science and inform the overall membership of the most striking advancements in their field. The workshops organized by the working groups continue to provide a wide range of topics of interest to all members of the Society.

We are thrilled you've elected to join us in Scottsdale and we look forward to your individual contributions to this conference.

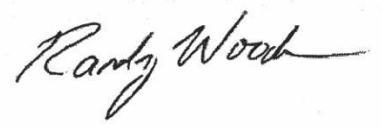

Randy Woodson

ASHS President 


\section{Conference Issue - ASHS 2007, Scottsdale, Ariz.}

Welcome letter from

ASHS President, William R. Woodson inside cover

Contents .749

General Information. .751

Conference-at-a-Glance .752

Working Group Activities .756

Meetings of ASHS Committees and Other Official Bodies of the Society. .759

Tours .760

Special Events. .760

Undergraduate and Graduate Student Activities 761

Meetings of Related Organizations 761

Site Map: room locations .763

Guide to Exhibitors .766

ASHS Awards Ceremony .769

Hall of Fame .770

Fellows .771

Career .775

Publication .778

Student
Thank Yous and Acknowledgements .782

Day-by Day Schedule

Monday .785

Tuesday 800

Wednesday 820

Thursday. .835

Friday 847

Abstracts (Oral Sessions)

Monday. 849

Tuesday. 861

Wednesday; 889

Thursday..... .905

Abstracts (Poster Sessions)

Monday..... 919

Tuesday. .942

Wednesday .970

Thursday .996

Index of Authors, Presenters, Meeting Chairs, and Moderators

Front cover: Arizona Sunset

Photo: Courtesy of Westin Kierland Resort and Spa-host hotel for ASHS-07.

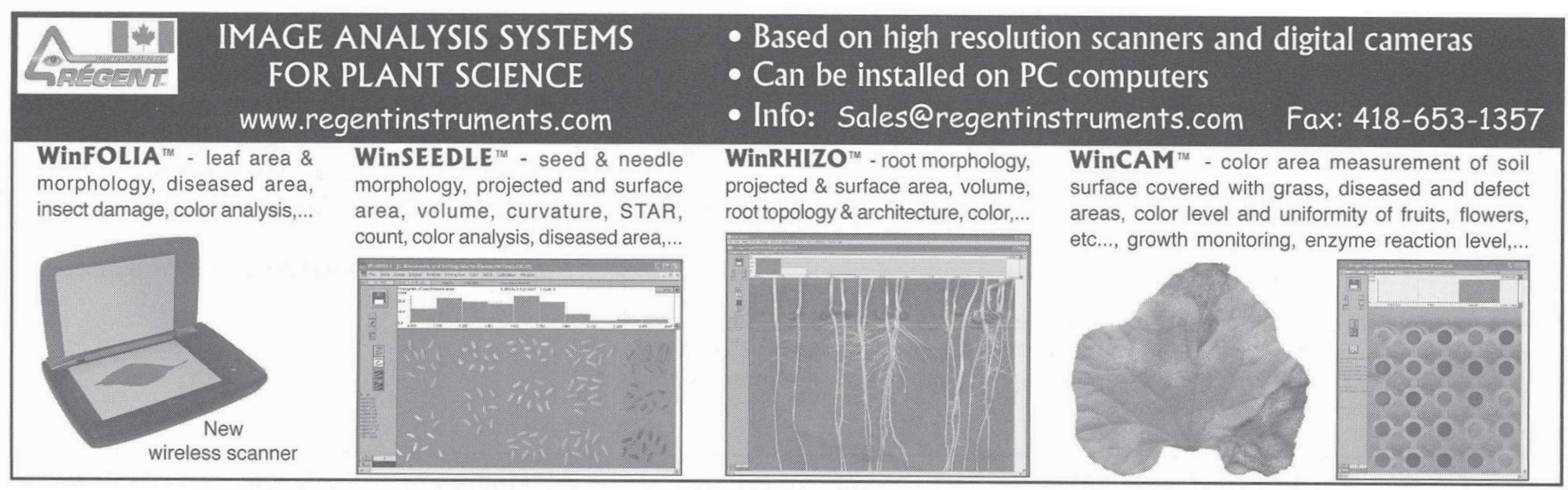


(ISSN 0018-5345)

July 2007

HortScience (ISSN 0018-5345) is a bimonthly publication of the American Society for Horticultural Science (ASHS), 113 South West Street, Suite 200, Alexandria, VA 223142851. Subscription rates: United States, Canada, and Mexico: $\$ 400$ per volume. All other countries: $\$ 450$ per volume (optional Airmail Delivery Service, $\$ 60.00$ additional). Periodicals postage is paid at Alexandria, Va., and at additional mailing offices. Postmaster: Please send address changes to HortScience, ASHS 113 South West Street, Suite 200, Alexandria, VA 22314-2851. Remittances should be made payable (in U.S. dollars) to American Society for Horticultural Science.

Manuscript submission. All initial contributions and correspondence should be submitted online at www.ashs.org. Other correspondence may be sent to the Publications Dept., ASHS, 113 South West Street, Suite 200, Alexandria, VA 22314-2851; phone +703.836.4606; fax +703.836.2024. Book reviews should be sent to Book Review Editor Allen V. Barker, Plant and Soil Sciences Dept., Bowditch Hall, Box 3091, Univ. of Massachusetts, Amherst, MA 01003-0910; 413-545-4733; fax 413-545-0260; e-mail barker@pssci.umass.edu.

Editorial production. Obituaries and correspondence regarding proofs of manuscripts in production should be sent to: Publications Dept., 113 South West Street, Suite 200, Alexandria, VA 22314-2851; phone +703.836.4606; fax +703.836.2024; e-mail hortscience@ashs.org.

Advertising. Inquiries and correspondence regarding advertising should be sent to HortScience Advertising, ASHS, 113 South West Street, Suite 200, Alexandria, VA 22314-2851; phone +703.836.4606; fax +703.836.2024; e-mail ashs@ ashs.org.

Business office. Correspondence regarding ASHS membership, subscriptions, replacement copies, claims, changes of address, publishing fees, reprints, and price and availability of back volumes and issues should be sent to Business Office, ASHS, 113 South West Street, Suite 200, Alexandria, VA22314-2851; phone+703.836.4606; fax +703.836.2024; e-mail ashs@ashs.org.

The American Society for Horticultural Science is not responsible for statements and opinions published in HortScience; they represent the views of the authors or persons to whom they are credited and are not necessarily those of the society. The publication of research information by the society does not constitute a recommendation or endorsement of products involved; for general use, label recommendations and directions must be followed.

Copyright $^{\circledR}$ 2007. American Society for Horticultural Science. All rights reserved.

Printed by The Sheridan Press, 450 Fame Ave., Hanover, PA 17331.

Board of Directors

*Chair. ..Paul E. Read

*President William R. Woodson

*President-elect.................................................................................. Mary M. Peet

Research Division Vice President ................................................................ John R. Clark

Education Division Vice President ............................................................ Janet C. Cole

Extension Division Vice President.................................................. Matthew D. Kleinhenz

Industry Division Vice President ....................................................... Craig A. Campbell

International Division Vice President ................................................... Richard J. Campbell

*Finance Committee Chair …………......................................................... Paul Smeal

*Publications Committee Chair ......................................................... Yin-Tung Wang

*Executive Committee

Publications Committee

Yin-Tung Wang

Caula Beyl

Louise Ferguson

Robert Joly

Carol Lovatt

Penelope Perkins-Veazie

John E. Preece

Marvin P. Pritts

Robert Trigiano

*Neal E. De Vos

*M. LeRon Robbins

*William R. Woodson

*Ex Officio

HortScience is covered in the following indexing and abstracting services: AGRICOLA; BIOBASE, Plant Science; BIOSIS, Biological Abstracts, BIOSIS Previews, basic BIOSIS; CABI, CAB Abstracts, CAB Direct, Horticultural Abstracts, Hort CD, Ornamental Horticulture, Postharvest News and Information, Review of Aromatic and Medicinal Plants, Review of Plant Pathology; Chemical Abstracts Service, CAPlus; ISI, Current Contents (Agriculture, Biology, and Environmental Science), Science Citation Index, Science Citation Index Expanded, Sci Search, ISI Alerting Service, Reference Update.

Mission Statement

HortScience publishes applied horticultural information of interest to a broad array of horticulturists. Its goals are to apprise horticultural scientists and others interested in horticulture of scientific and industry developments and of significant research, education, or extension findings or methods.
Editor in Chief

M. LeRon Robbins

Publisher

Michael W. Neff

Consulting Editors

Breeding, Cultivars, Rootstocks, and Germplasm

Resources

Fruit

Adam Dale

Vegetables and ornamentals

Paul R. Fantz

Raymond J. Schnell

Jay Scott

Crop Production

Floricultural plants

Grapes and berries

David Bryla

Nursery and landscape plants

Subtropical and tropical fruit

Thomas E. Marler

Temperate fruit and tree nuts

Robert Ebel

Anand K. Yadav

Vegetables

Clinton Shock

General Horticulture

Robert L. Morris

Growth Regulators (applications)

Marketing and Economics

Robin G. Brumfield

Postharvest Biology and Technology

Ornamentals

Peter Sholberg

Temperate Fruit

Penelope Perkins-Veazie

Vegetables

Jeffrey K. Brecht

Propagation and Tissue Culture

Paula M. Pijut

Seed Technology

Alan Taylor

Soil Management, Fertilization, and Irrigation

Charles A. Sanchez

Statistics

George Fernandez

Turf Management

David Aldous

Erik H. Ervin

Barry Stewart

Colloquia, Workshops, and Feature Articles

Steven F. Vaughn

Book Reviews

Allen V. Barker

Register of New Fruit and Nut Varieties

John Clark

Chad Finn

Vegetable Cultivar Descriptions for North America Todd C. Wehner 


\section{General Information}

\section{Welcome to Scottsdale, Arizona!}

The American Society for Horticultural Science (ASHS) welcomes you to the 104th Annual Conference at the Westin Kierland Resort and Spa, Scottsdale, Ariz. ASHS has prepared an exciting package of special events for attendees to experience the endless variety of activities in and surrounding Scottsdale.

\section{Emergencies}

Police/Medical

Call 911

\section{Conference Facilities}

All conference activities, unless noted otherwise, will take place at the Westin Kierland Resort and Spa. Please check the detailed program and schedule for exact room locations. The registration desk and placement service office will be open starting Monday, 16 July 2007.

\section{Registration}

The ASHS-2007 registration desk will be located at the Culturekeepers Hall West. The hours of operation are:

$\begin{array}{ll}\text { Monday, July } 16 & 7: 30 \mathrm{am}-6: 00 \mathrm{pm} \\ \text { Tuesday, July } 17 & 7: 30 \mathrm{am}-6: 00 \mathrm{pm} \\ \text { Wednesday, July 18 } & 7: 30 \mathrm{am}-5: 30 \mathrm{pm} \\ \text { Thursday, July } 19 & 8: 00 \mathrm{am}-4: 00 \mathrm{pm}\end{array}$

\section{Message Center/E-mail Stations}

Messages for attendees may be posted throughout the meeting on the Message Board. E-mail stations are available during the following times:

$\begin{array}{ll}\text { Monday, July } 16 & 12: 00 \mathrm{pm}-6: 00 \mathrm{pm} \\ \text { Tuesday, July } 17 & 9: 00 \mathrm{am}-6: 00 \mathrm{pm} \\ \text { Wednesday, July } 18 & 9: 00 \mathrm{am}-5: 30 \mathrm{pm} \\ \text { Thursday, July } 19 & 9: 00 \mathrm{am}-2: 00 \mathrm{pm}\end{array}$

\section{Posters}

Posters must be hung in the Kierland Grand Ballroom, on Monday between the hours of 7:00 am and noon. Viewing hours are:

$\begin{array}{ll}\text { Monday, July } 16 & 12: 00 \mathrm{pm}-6: 00 \mathrm{pm} \\ \text { Tuesday, July } 17 & 9: 00 \mathrm{am}-6: 00 \mathrm{pm} \\ \text { Wednesday, July } 18 & 9: 00 \mathrm{am}-5: 30 \mathrm{pm} \\ \text { Thursday, July } 19 & 9: 00 \mathrm{am}-2: 00 \mathrm{pm}\end{array}$

Posters may be removed on Thursday, 19 July, after 2:00 pm

\section{Exhibits}

The Exposition includes suppliers of scientific, classroom, and greenhouse equipment; manufacturers of machinery used in the production and processing of horticultural crops; distributors of chemicals such as growth regulators, fungicides, and fertilizers; and publishers of horticultural and plant science publications. The exhibits are located in the Kierland Grand Ballroom, convenient to the technical sessions and other meetings. Exhibit hours are:

Setup:

Monday, July 16

8:00 am-12:00 pm

Exhibit Hours:

Monday, July 16

Tuesday, July 17

Wednesday, July 18

Thursday, July 19

Dismantling:

Thursday, July 19

\author{
12:00 pm-6:00 pm \\ 9:00 am-6:00 pm \\ 9:00 am-5:30 pm \\ 9:00 am-2:00 pm \\ 2:00 pm-5:00 pm
}

\section{Placement/Job Internship Service}

To participate in the ASHS Placement Service during the 2007 Annual Conference, individuals looking for positions and/or organizations with positions available should fill out and submit copies of the appropriate forms. Candidates also may submit resumes to be on file at the Placement Service. The Placement Service will be located in Cushing A/B and will be open during the following hours:

$\begin{array}{ll}\text { Monday, July } 16 & 12: 00 \mathrm{pm}-6: 00 \mathrm{pm} \\ \text { Tuesday, July } 17 & 8: 00 \mathrm{am}-6: 00 \mathrm{pm} \\ \text { Wednesday, July } 18 & 8: 00 \mathrm{am}-5: 30 \mathrm{pm} \\ \text { Thursday, July } 19 & 8: 00 \mathrm{am}-4: 00 \mathrm{pm}\end{array}$

\section{Press and Member Information}

The ASHS staff will be available at Registration. Members of the press may obtain further information on ASHS programs and activities during the following hours:

$\begin{array}{ll}\text { Monday } & 7: 30 \mathrm{am}-6: 00 \mathrm{pm} \\ \text { Tuesday } & 7: 30 \mathrm{am}-6: 00 \mathrm{pm} \\ \text { Wednesday } & 7: 30 \mathrm{am}-5: 30 \mathrm{pm} \\ \text { Thursday } & 8: 00 \mathrm{am}-4: 00 \mathrm{pm}\end{array}$

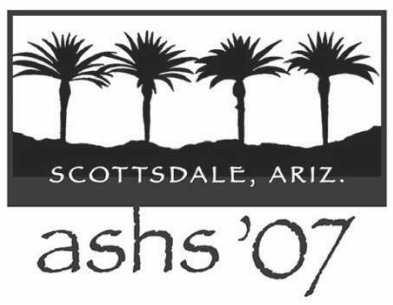

Please Note:

All information in regard to program and abstract scheduling for the ASHS2007 Annual Conference is accurate and up-to-date as of the time this issue of HortScience goes to press. During the Conference, check the message boards and at the registration desk for any updates. 
CONFERENCE AT A GLANCE (subject to change)

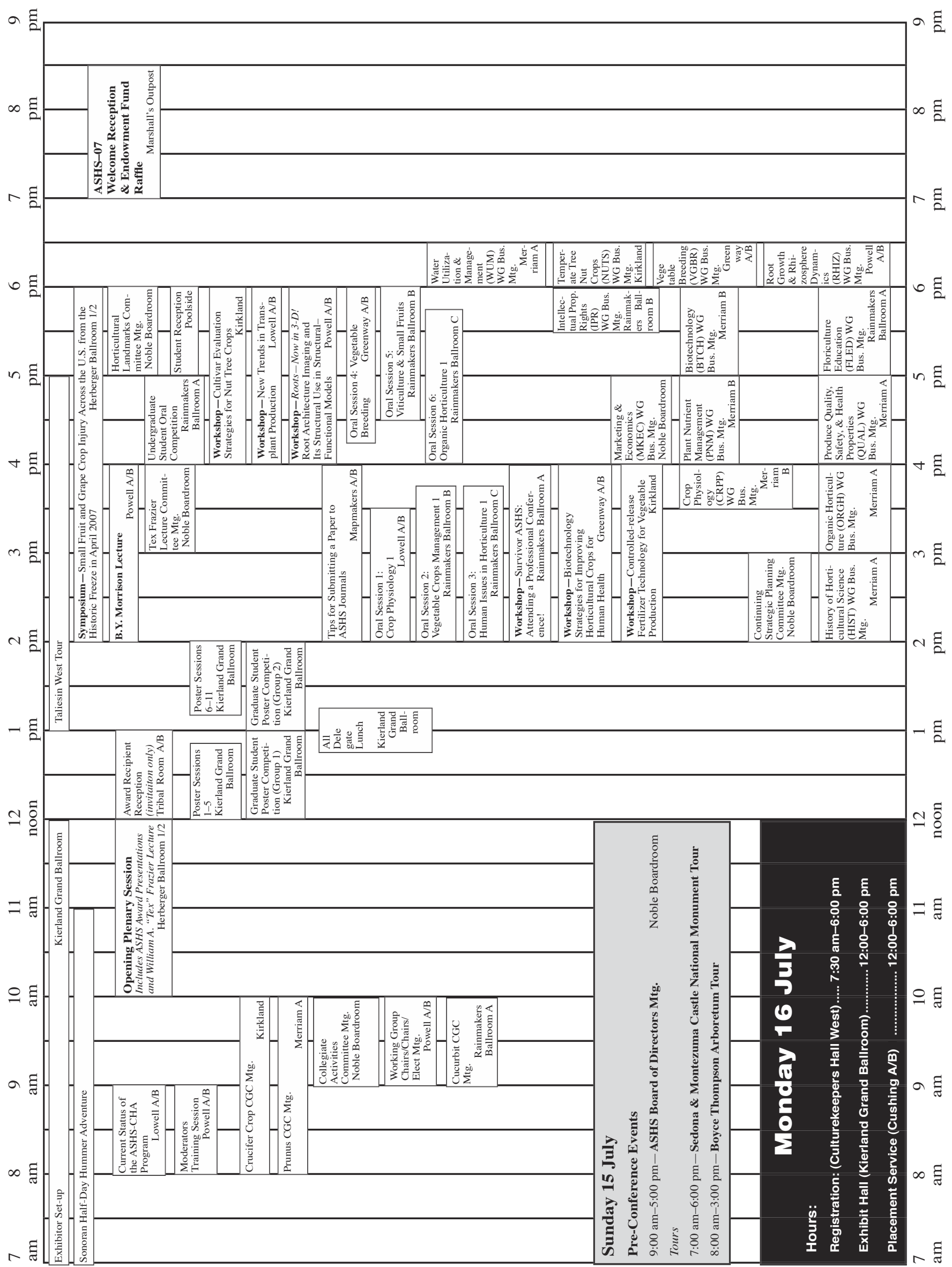




\section{CONFERENCE AT A GLANCE (subject to change)}

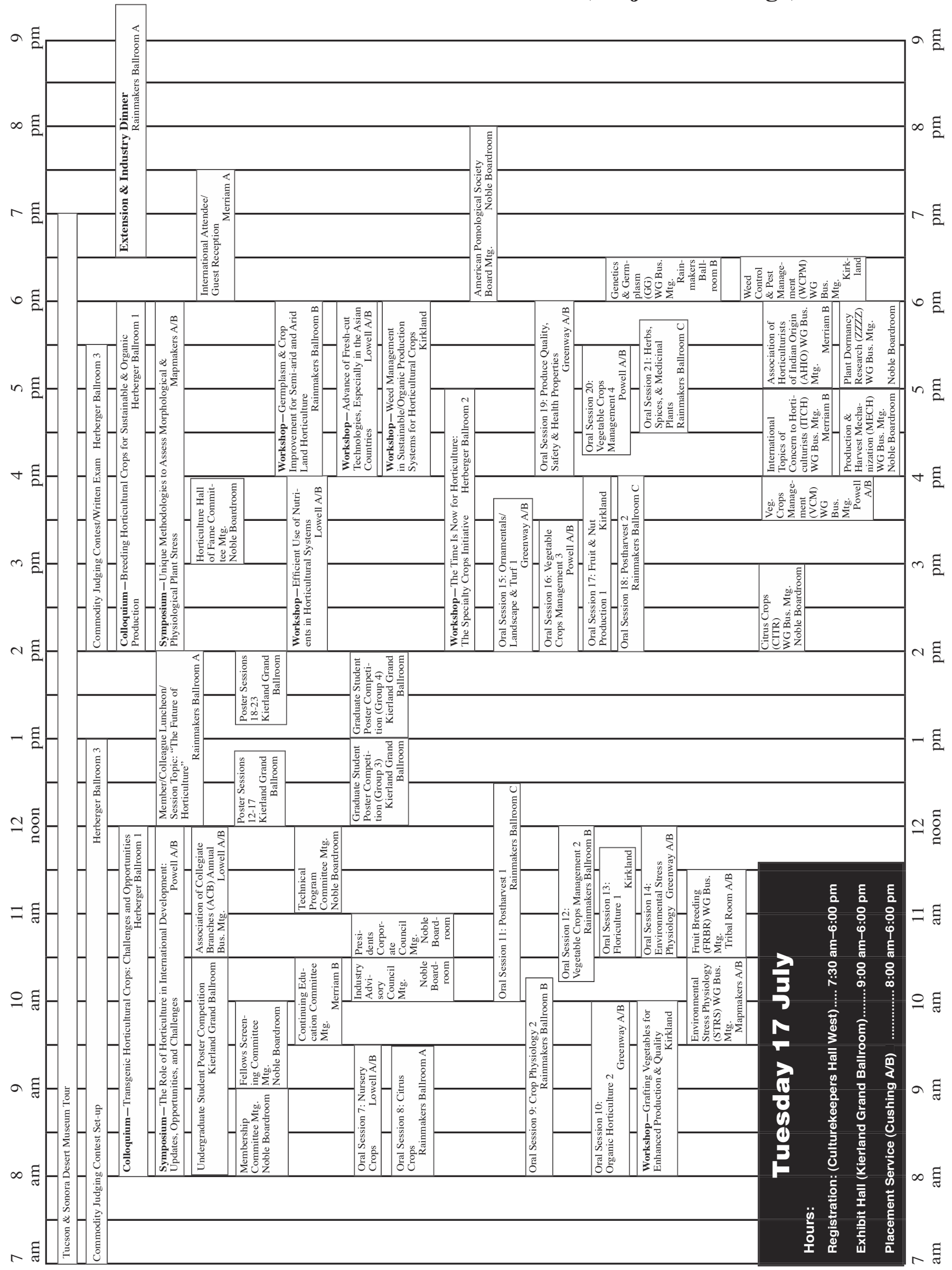




\section{CONFERENCE AT A GLANCE (subject to change)}

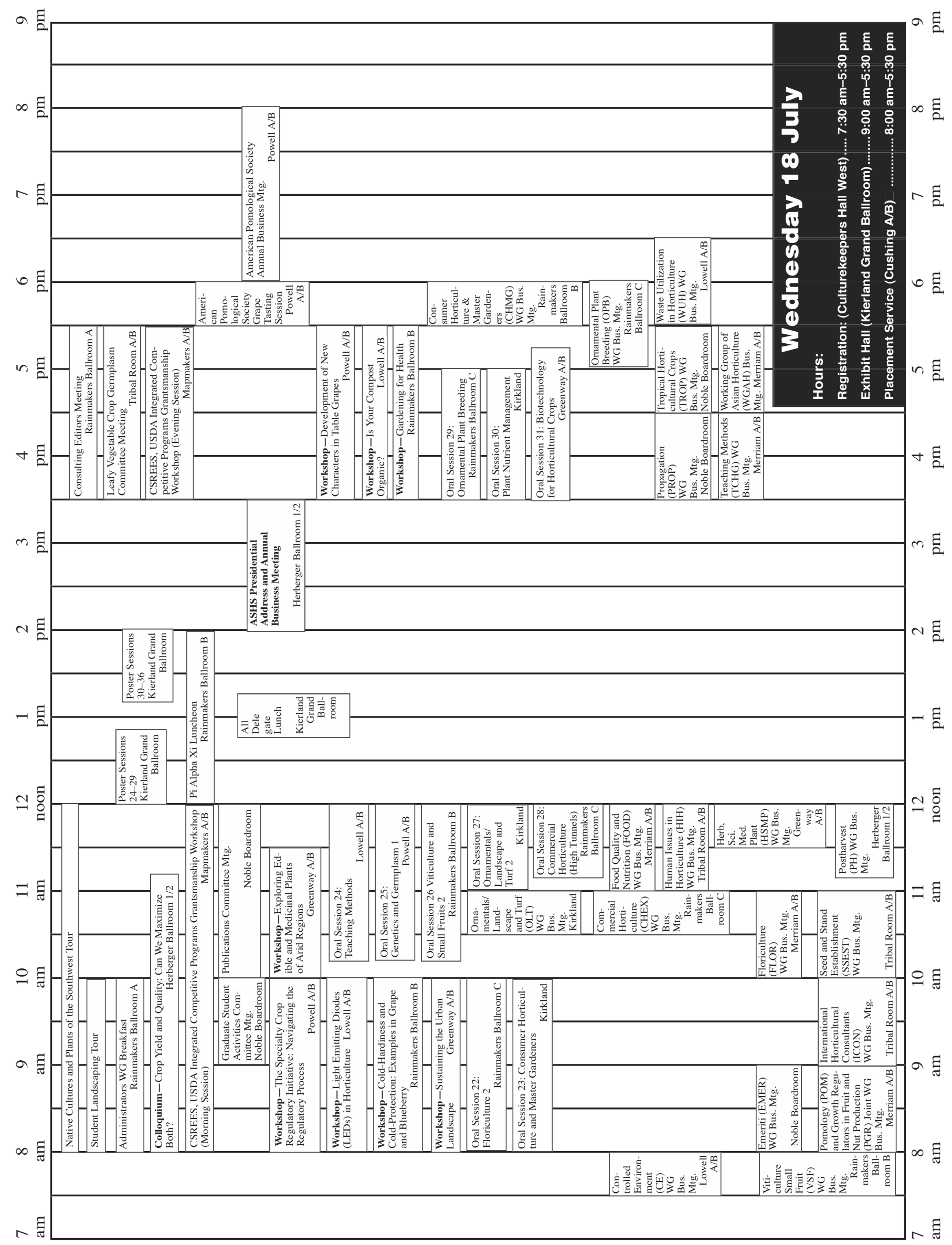


CONFERENCE AT A GLANCE (subject to change)

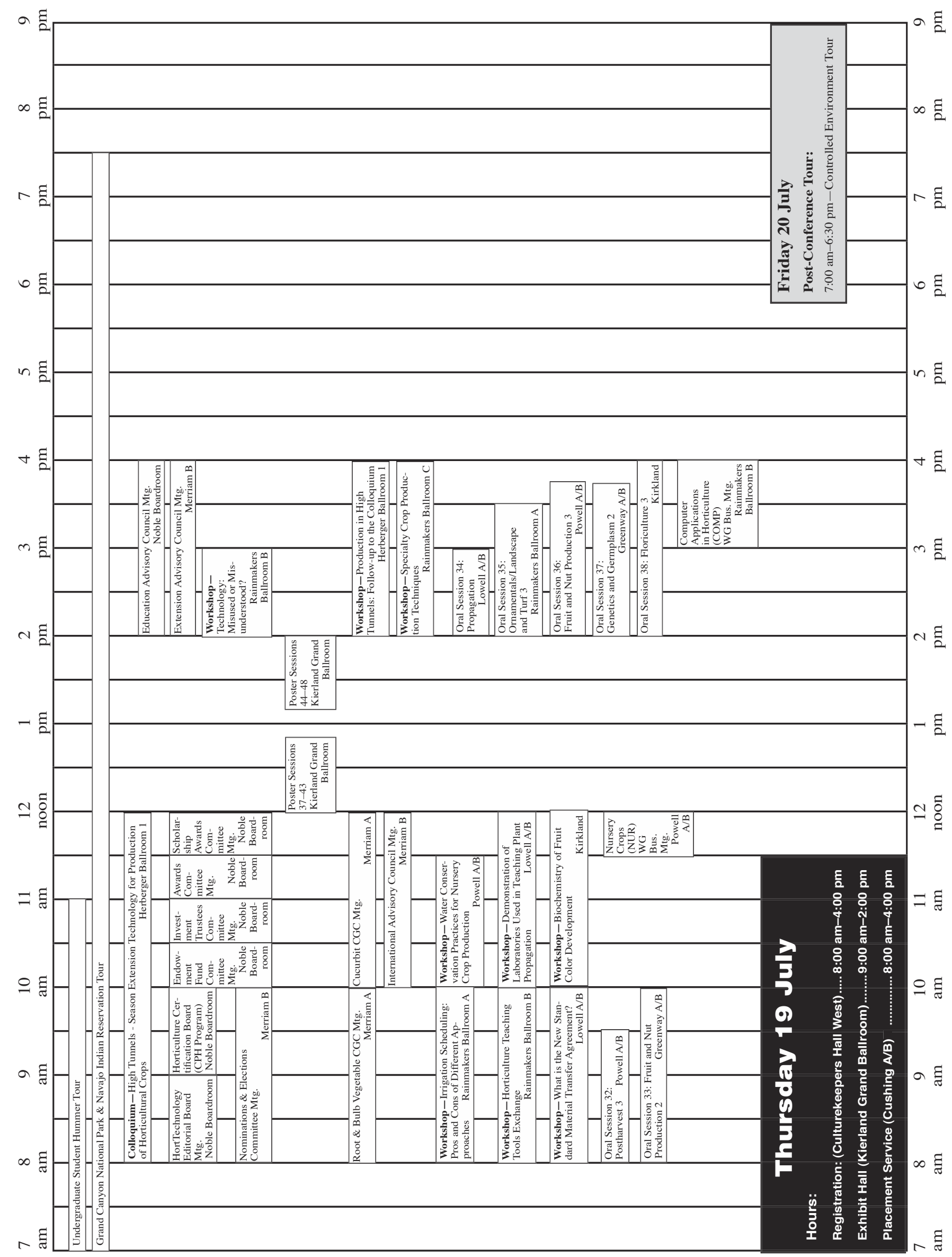




\section{ASHS WORKING GROUP ACTIVITIES (subject to change)}

\section{RESEARCH DIVISION (commodity)}

Citrus Crops (CITR)

Business Meeting

Crop Physiology (CRPP)

Business Meeting

Workshop-Irrigation Scheduling: Pros and Cons of Different Approaches

Floriculture (FLOR)

Business Meeting

Fruit Breeding (FRBR)

Business Meeting

Colloquium - Breeding Horticultural Crops for Sustainable and Organic Production

Growth Regulators in Fruit and Nut Production (PGR)

Business Meeting

Herbs, Spices and Medicinal Plants (HSMP)

Workshop-Exploring Edible and Medicinal Plants of Arid Regions

Business Meeting

Nursery Crops (NUR)

Workshop-Water Conservation Practices for Nursery Crop Production

Business Meeting

Ornamentals/Landscape and Turf (O/LT)

Workshop-Sustaining the Urban Landscape

Business Meeting

Ornamental Plant Breeding (OPB)

Colloquium - Breeding Horticultural Crops for Sustainable and Organic Production

Business Meeting

Pomology (POM)

Business Meeting

Workshop-Biochemistry of Fruit Color Development

Temperate Tree Nut Crops (NUTS)

Workshop-Cultivar Evaluation Strategies for Nut Tree Crops

Business Meeting

Vegetable Breeding (VGBR)

Business Meeting

Colloquium - Breeding Horticultural Crops for Sustainable and Organic Production

Vegetable Crops Management (VCM)

Workshop-Controlled-release Fertilizer Technology for Vegetable Production

Colloquium - Breeding Horticultural Crops for Sustainable and Organic Production

Business Meeting

Workshop-Specialty Crop Production Techniques

Viticulture and Small Fruits (VSF)

Business Meeting

Workshop-Cold-Hardiness and Cold-Protection: Examples in Grape and Blueberry

\begin{tabular}{|c|c|}
\hline Tues., 17 July & $2: 00-3: 00 \mathrm{pm}$ \\
\hline Mon., 16 July & $3: 30-4: 00 \mathrm{pm}$ \\
\hline Thurs., 19 July & 8:00-10:00 am \\
\hline Wed., 18 July & 10:00-11:00 am \\
\hline Tues., 17 July & 10:30:00-11:30 am \\
\hline Tues., 17 July & $2: 00-6: 00 \mathrm{pm}$ \\
\hline Wed., 18 July & 8:00-10:00 am \\
\hline $\begin{array}{l}\text { Wed., } 18 \text { July } \\
\text { Wed., } 18 \text { July }\end{array}$ & $\begin{array}{l}10: 00-11: 30 \mathrm{am} \\
11: 30 \mathrm{am}-12: 00 \mathrm{pm}\end{array}$ \\
\hline $\begin{array}{l}\text { Thurs., } 19 \text { July } \\
\text { Thurs., } 19 \text { July }\end{array}$ & $\begin{array}{l}10: 00-11: 30 \mathrm{am} \\
11: 30 \mathrm{am}-12: 00 \mathrm{pm}\end{array}$ \\
\hline $\begin{array}{l}\text { Wed., } 18 \text { July } \\
\text { Wed., } 18 \text { July }\end{array}$ & $\begin{array}{l}8: 00-10: 00 \mathrm{am} \\
10: 30-11: 00 \mathrm{am}\end{array}$ \\
\hline $\begin{array}{l}\text { Tues., } 17 \text { July } \\
\text { Wed., } 18 \text { July }\end{array}$ & $\begin{array}{l}\text { 2:00-6:00 pm } \\
5: 00-6: 00 \mathrm{pm}\end{array}$ \\
\hline $\begin{array}{l}\text { Wed., } 18 \text { July } \\
\text { Thurs., } 19 \text { July }\end{array}$ & $\begin{array}{l}8: 00-10: 00 \mathrm{am} \\
10: 00 \mathrm{am}-12: 00 \mathrm{pm}\end{array}$ \\
\hline $\begin{array}{l}\text { Mon., } 16 \text { July } \\
\text { Mon., } 16 \text { July }\end{array}$ & $\begin{array}{l}\text { 4:00-6:00 pm } \\
\text { 6:00-6:30 pm }\end{array}$ \\
\hline Mon., 16 July & $6: 00-6: 30 \mathrm{pm}$ \\
\hline Tues., 17 July & $2: 00-6: 00 \mathrm{pm}$ \\
\hline Mon., 16 July & $2: 00-4: 00 \mathrm{pm}$ \\
\hline $\begin{array}{l}\text { Tues., } 17 \text { July } \\
\text { Tues., } 17 \text { July } \\
\text { Thurs., } 19 \text { July }\end{array}$ & $\begin{array}{l}2: 00-6: 00 \mathrm{pm} \\
3: 30-4: 00 \mathrm{pm} \\
2: 00-4: 00 \mathrm{pm}\end{array}$ \\
\hline Wed., 18 July & $7: 30-8: 00 \mathrm{am}$ \\
\hline Wed., 18 July & 8:00-10:00 am \\
\hline
\end{tabular}

Noble Boardroom

Merriam B

Rainmakers Ballroom A

Merriam A/B

Tribal Room A/B

Herberger Ballroom 1

Merriam A/B

Greenway A/B

Greenway A/B

Powell A/B

Powell A/B

Greenway A/B

Kirkland

Herberger Ballroom 1 Rainmakers Ballroom C

Merriam A/B

Kirkland

Kirkland

Kirkland

Greenway A/B

Herberger Ballroom 1

Kirkland

Herberger Ballroom 1

Powell A/B

Rainmakers Ballroom C

Rainmakers Ballroom B

Rainmakers Ballroom B

\section{RESEARCH DIVISION (cross-commodity)}

Environmental Stress Physiology (STRS)

Business Meeting

Symposium - Unique Methodologies to Assess Morphological and Physiological Plant Stress

\author{
Tues., 17 July \\ 9:30-10:30 am \\ Tues., 17 July \\ 2:00-6:00 pm
}

Mapmakers A/B

Mapmakers A/B 
Genetics and Germplasm (GG)

Colloquium-Transgenic Horticultural Crops: Challenges and Opportunities

Workshop-Germplasm and Crop Improvement for Semi-arid and Arid Land Horticulture

Business Meeting

Workshop-The Specialty Crop Regulatory Initiative: Navigating the Regulatory Process

Growth Chambers and Controlled Environments (CE)

Workshop-Light Emitting Diodes (LEDs) in Horticulture

Business Meeting

Human Issues in Horticulture (HIH)

Business Meeting

Workshop-Gardening for Health

Organic Horticulture (ORGH)

Business Meeting

Colloquium-Breeding Horticultural Crops for Sustainable and Organic Production

Workshop-Gardening for Health
Tues., 17 July

Tues., 17 July

Tues., 17 July

Wed., 18 July

Wed., 18 July

Wed., 18 July

Wed., 18 July

Wed., 18 July

Mon., 16 July

Tues., 17 July

Wed., 18 July

Mon., 16 July

Mon., 16 July

Tues., 17 July

Mon., 16 July

Tues., 17 July

Tues., 17 July

Wed., 18 July

Wed., 18 July

Mon., 16 July

Tues., 17 July

Tues., 17 July

Wed., 18 July

Thurs., 19 July

Mon., 16 July

Mon., 16 July

Mon., 16 July

Wed., 18 July

Wed., 18 July

Mon., 16 July

Tues., 17 July
8:00 am-12:00 pm

4:00-6:00 pm

6:00-6:30 pm

8:00-10:00 am

8:00-10:00 am

7:30-8:00 am

11:00 am-12:00 pm

3:30-5:30 am

3:00-4:00 pm

2:00-6:00 pm

$3: 30-5: 30$ am

2:00-4:00 pm

5:00-6:00 pm

5:00-6:00 pm

4:00-5:00 pm

2:00-4:00 pm

4:00-6:00 pm

8:00-11:15 am

11:15 am-12:00 pm

4:00-5:00 pm

8:00-10:00 am

4:00-5:00 pm

$3: 30-4: 30 \mathrm{pm}$

10:00 am-12:00 pm

4:00-6:00 pm

6:00-6:30 pm

4:00-6:00 pm

10:00-11:00 am

Waste Utilization in Horticulture (WUH)

Business Meeting

Water Utilization and Management (WUM)

Business Meeting

Weed Control and Pest Management (WCPM)

Business Meeting
5:30-6:00 pm

6:00-6:30 pm

6:00-6:30 pm
Herberger Ballroom 1

Rainmakers Ballroom B Rainmakers Ballroom B

Powell A/B

Lowell A/B

Lowell A/B

Tribal Room A/B

Rainmakers Ballroom B

Merriam A

Herberger Ballroom 1 Rainmakers Ballroom B

Greenway A/B Merriam B

Noble Boardroom

Merriam B

Lowell A/B

Lowell A/B

Herberger Ballroom 1/2 Herberger Ballroom 1/2

Merriam A

Kirkland Noble Boardroom

Noble Boardroom

Lowell A/B

Powell A/B Powell A/B

Lowell A/B Tribal Room A/B

Lowell A/B

Merriam A

Kirkland 


\section{EDUCATION DIVISION}

General

Education Advisory Council

Administrators (ADM)

Working Group Breakfast

Computer Applications in Horticulture (COMP)

Workshop-Technology: Misused or Misunderstood?

Business Meeting

Emeriti (EMER)

Business Meeting

Floriculture Education (FLED)

Business Meeting

History of Horticultural Science (HIST)

Business Meeting

Teaching Methods (TCHG)

Business Meeting

Workshop-Horticulture Teaching Tools Exchange

$\begin{array}{lcr}\text { Thurs., } 19 \text { July } & \text { 2:00-4:00 pm } & \text { Noble Boardroom } \\ \text { Wed., 18 July } & 8: 00-10: 00 \mathrm{am} & \text { Rainmakers Ballroom A } \\ \text { Thurs., 19 July } & 2: 00-3: 00 \mathrm{pm} & \text { Rainmakers Ballroom B } \\ \text { Thurs., 19 July } & 3: 00-4: 00 \mathrm{pm} & \text { Rainmakers Ballroom B } \\ \text { Wed., 18 July } & 8: 00-9: 00 \mathrm{am} & \text { Noble Boardroom } \\ & & \\ \text { Mon, 16 July } & 5: 00-6: 00 \mathrm{pm} & \text { Rainmakers Ballroom A } \\ \text { Mon, 16 July } & 2: 00-3: 00 \mathrm{pm} & \text { Merriam A } \\ & & \\ \text { Wed., 18 July } & 3: 30-4: 30 \mathrm{pm} & \text { Merriam A/B } \\ \text { Thurs., 19 July } & 8: 00-10: 0 \mathrm{am} & \end{array}$

EXTENSION DIVISION

\section{General}

Extension and Industry Dinner

Extension Advisory Council

Tues., 17 July

Thurs., 19 July

6:30-10:00 pm

2:00-4:00 pm

Tues., 17 July

2:00-6:00 pm

Wed., 18 July

10:30-11:00 am

Thurs., 19 July

8:00 am-12:00 pm

Thurs., 19 July

2:00-4:00 pm

Wed., 18 July

Wed., 18 July
$3: 30-5: 30 \mathrm{am}$

5:30-6:00 pm
Rainmakers Ballroom A Merriam B

Herberger Ballroom 1 Rainmakers Ballroom C

Herberger Ballroom 1

Herberger Ballroom 1

Rainmakers Ballroom B Rainmakers Ballroom B

\section{INDUSTRY DIVISION}

\section{General}

Extension and Industry Dinner

Industry Advisory Council

President's Corporate Council

\section{Intellectual Property Rights (IPR)}

Business Meeting

Workshop-What is the New Standard Material Transfer Agreement?

Marketing and Economics (MKEC)

Business Meeting

Workshop-The Specialty Crop Regulatory Initiative: Navigating the Regulatory Process

$\begin{array}{ll}\text { Tues., 17 July } & 6: 30-10: 00 \mathrm{pm} \\ \text { Tues., 17 July } & \text { 10:00-10:30 am } \\ \text { Tues., 17 July } & \text { 10:30-11:00 am } \\ \text { Mon., 16 July } & \text { 5:30-6:00 pm } \\ \text { Thurs., 19 July } & 8: 00-10: 00 \mathrm{am} \\ \text { Mon., 16 July } & \text { 4:00-5:00 pm } \\ \text { Wed., 18 July } & 8: 00-10: 00 \mathrm{am}\end{array}$

Rainmakers Ballroom A Noble Boardroom Noble Boardroom

Rainmakers Ballroom B

Lowell A/B

Noble Boardroom

Powell A/B

\section{INTERNATIONAL DIVISION}

\section{General}

International Division Reception

International Advisory Council

\section{Association of Horticulturists of Indian Origin (AHIO)}

Business Meeting

International Horticultural Consultants (ICON)

Symposium - The Role of Horticulture in International

Development: Updates, Opportunities and Challenges Business Meeting

\author{
Tues., 17 July 6:00-7:30 pm \\ Thurs., 19 July 10:00 am-12:00 pm \\ Tues., 17 July 5:00-6:00 pm \\ 8:00 am-12:00 pm \\ 9:00-10:00 am
}

Merriam A

Merriam B

Merriam B

Powell A/B

Tribal Room A/B 
International Topics of Concern (ITCH)

Symposium - The Role of Horticulture in International

Development: Updates, Opportunities and Challenges

Business Meeting

Tues., 17 July

Tues., 17 July

8:00 am-12:00 pm

4:00-5:00 pm

Wed., 18 July

$4: 30-5: 30 \mathrm{pm}$

Tues., 17 July

Wed., 18 July
Powell A/B

Merriam B

Noble Boardroom

Lowell A/B Merriam A/B

\section{MEETINGS OF ASHS COMMITTEES AND OTHER OFFICIAL BODIES OF THE SOCIETY}

\section{Sunday, 15 July}

ASHS Board of Directors

\section{Monday, 16 July}

Working Group Chairs and Chairs-elect

Collegiate Activities Committee

Continuing Strategic Planning Committee

Tex Frazier Lecture Committee

Horticultural Landmarks Committee

\section{Tuesday, 17 July}

Membership Committee

Fellows Committee

Continuing Education Committee

Technical Program Committee

Horticulture Hall of Fame Selection Committee

\section{Wednesday, 18 July}

Graduate Student Activities Committee

Publications Commmittee

\section{Thursday, 19 July}

Nominations \& Elections Committee

Endowment Fund Committee

Investment Trustees Committee

Awards Committee

Scholarship Awards Committee
9:00 am-5:00 pm

Noble Boardroom

9:00-10:00 am
9:00-10:00 am
2:00-3:00 pm
3:00-4:00 pm
5:00-6:00 pm

8:00-9:00 am

9:00-10:00 am

9:30-10:30 am

11:00 am-12:00 pm

3:00-4:00 pm

9:00-10:00 am

10:00 am-12:00 pm

8:00-10:00 am

10:00-10:30 am

10:30-11:00 am

11:00-11:30 am

11:30 am-12:00 pm
Powell A/B

Noble Boardroom

Noble Boardroom

Noble Boardroom

Noble Boardroom

Noble Boardroom

Noble Boardroom

Merriam B

Noble Boardroom

Noble Boardroom

Noble Boardroom

Noble Boardroom

Merriam B

Noble Boardroom

Noble Boardroom

Noble Boardroom

Noble Boardroom 


\section{TOURS}

\section{Sunday, 15 July}

7:00 am-6:00 pm

8:00 am-3:00 pm

Monday, 16 July

7:00-11:00 am

1:00-5:00 pm

\section{Tuesday, 17 July}

7:00 am-7:30 pm

Wednesday, 18 July

8:00 am-12:00 pm

8:00 am-12:00 pm

Thursday, 19 July

7:00-11:00 am

7:00 am-7:00 pm

Friday, 20 July

7:00 am-6:30 pm
Sedona \& Montezuma Castle National Monument Tour

Boyce Thompson Arboretum Tour

Sonoran Half-Day Hummer Adventure

Taliesin West

Tucson \& Sonora Desert Museum Tour
Native Cultures and Plants of the Southwest Tour

Student Landscaping Tour

Undergraduate Student Hummer Tour

Grand Canyon National Park \& Navajo Indian Reservation Tour

Controlled Environment Tour

\section{SPECIAL EVENTS}

\section{Monday, 16 July}

8:00-9:00 am

Current Status of the ASHS Certified Horticultural Advisor Program

Lowell A/B

10:00 am-12:00 pm

2:00-4:00 pm

Opening Plenary Session, ASHS 2007 Awards Ceremony, and William A. "Tex" Frazier Lecture

Herberger Ballroom 1/2

B.Y. Morrison Lecture

Tips for Submitting a Paper to ASHS Journals

Welcome Reception and Endowment Fund Raffle

Powell A/B

Mapmakers A/B

7:00-8:30 pm

Tuesday, 17 July

12:00-2:00 pm

6:00-7:00 pm

6:30-10:00 pm

Member/Colleague Luncheon/Session-

Topic: "The Future of Horticulture"

International Attendee/Guest Reception

Rainmakers Ballroom A

Merriam A

Extension/Industry Reception \& Dinner

Rainmakers Ballroom A

Wednesday, 18 July

8:00 am-12:00 pm

CSREES, USDA Integrated Competitive Programs Grantsmanship Workshop (Morning Session)

Mapmakers A/B

2:00-3:30 pm

ASHS Presidential Address and Annual Business Meeting

$3: 30-5: 30 \mathrm{pm}$

CSREES, USDA Integrated Competitive Programs

Grantsmanship Workshop (Afternoon Session)

Mapmakers A/B 


\section{UNDERGRADUATE AND GRADUATE STUDENT ACTIVITIES}

UNDERGRADUATE

\section{Monday 16 July}

2:00-4:00 pm

4:00-5:00 pm

5:00-6:00 pm

Tuesday, 17 July

7:00 am-1:00 pm

8:00-10:30 am

10:30 am-12:00 pm

2:00-5:30 pm

Wednesday, 18 July

8:00-10:00 am

Thursday, 19 July

7:00-11:00 am

Student Tour (ticketed)
Rainmakers Ballroom A

Rainmakers Ballroom A

Poolside

ACB Commodity Judging Contest Set-Up

Herberger Ballroom 3

Undergraduate Student Poster Competition

Kierland Grand Ballroom

Lowell A/B

ACB Commodity Judging Contest (Oral and Written)

Herberger Ballroom 3
Student Landscaping Tour (ticketed)

Westin Kierland Resort and Spa

GRADUATE Monday, 16 July

12:00-1:15 pm

Graduate Student Poster Competition, Group 1

Graduate Student Poster Competition, Group 2

\section{Tuesday, 17 July}

12:00-1:15 pm

Graduate Student Poster Competition, Group 3

$1: 15-2: 00 \mathrm{pm}$
Graduate Student Poster Competition, Group 4
Kierland Grand Ballroom

Kierland Grand Ballroom-

Kierland Grand Ballroom

Kierland Grand Ballroom

\section{MEETINGS OF RELATED ORGANIZATIONS}

\section{Monday, 16 July}

8:00-10:00 am

8:00-10:00 am

9:00-10:00 am

Wednesday, 18 July

12:00-2:00 pm

$3: 30-5: 30 \mathrm{pm}$

$5: 30-6: 00 \mathrm{pm}$

6:00-8:00 pm

Thursday, 19 July

8:00-10:00 am
Crucifer Crop Germplasm Committee

Prunus Crop Germplasm Committee

Cucurbit Genetics Cooperative

Pi Alpha Xi Luncheon

Leafy Vegetable Crop Germplasm Committee

American Pomological Society Grape Tasting Session

American Pomological Society Annual Business Meeting

Root and Bulb Vegetable Crop Germplasm Committee
Kirkland

Merriam A

Rainmakers Ballroom A

Rainmakers Ballroom B

Tribal Room A/B

Powell A/B

Powell A/B

Merriam A 


\section{Frst in its Field}

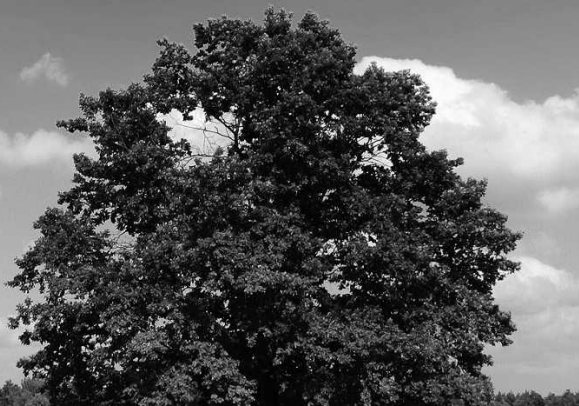

LI-COR's LI-6400 leads the way as the premiere portable photosynthesis system. Continuously developed and enhanced since 1995 to meet demanding research needs, LI-6400 design firsts include -

- IRGAs in the sensor head

- User cleanable optics

- Leaf chamber fluorometer

- Red/blue LED light source

- Remote Internet access NEW!

- Customer training program

- Real-time graphics

- Auto programs

- Multiple chamber options

The most referenced photosynthesis system in the world.

See the LI-6400 on the Web at

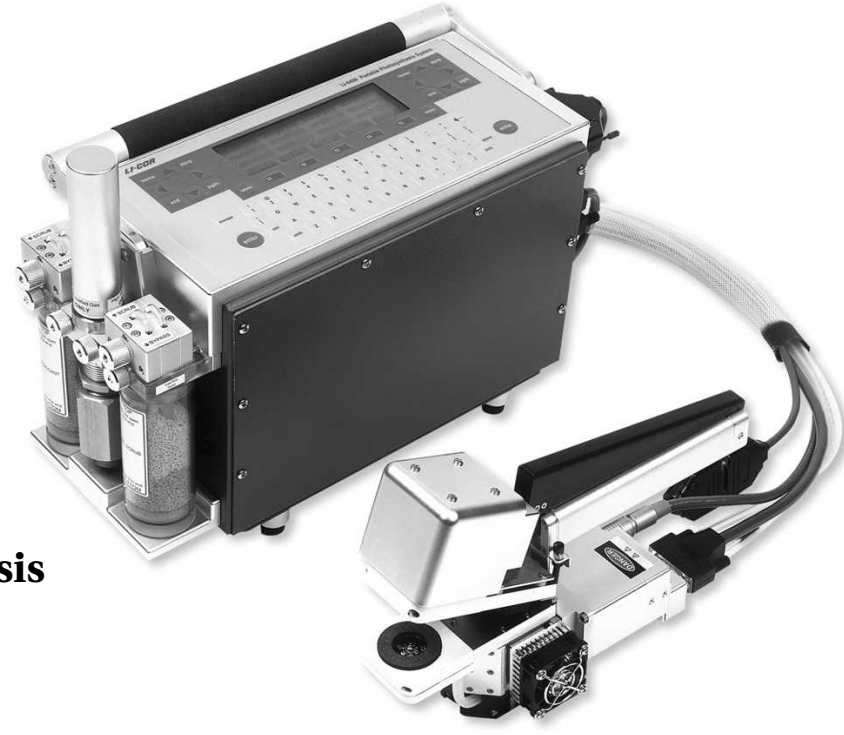
www.licor.com/6400

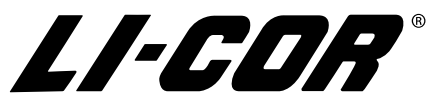

Biosciences

800-447-3576

LI-6400 is covered by patents pending. (C) 2006 LI-COR Biosciences

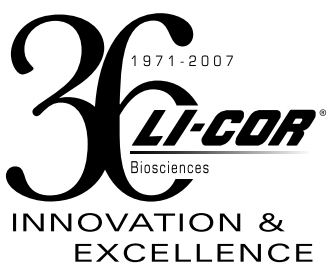




\section{American Society for Horticultural Science 2007 Annual Conference Floor Plan (site map)}

\section{Level 1, Lower Level}

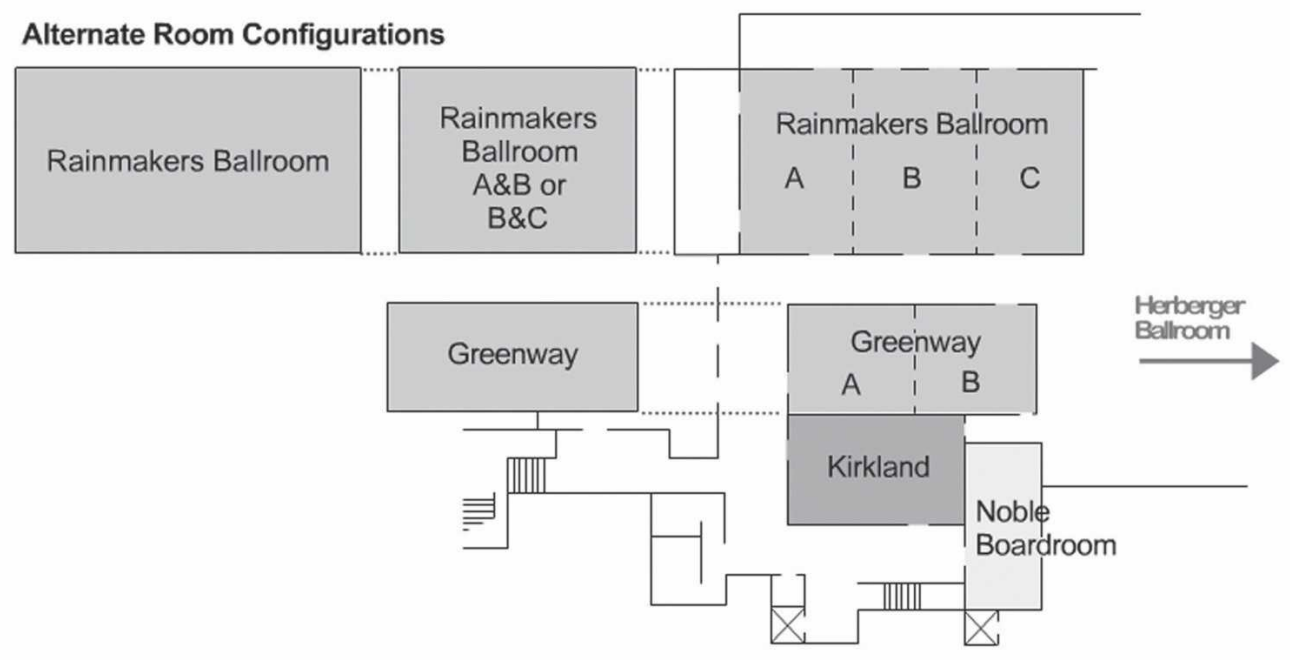

\section{Level 2, Lobby Level}

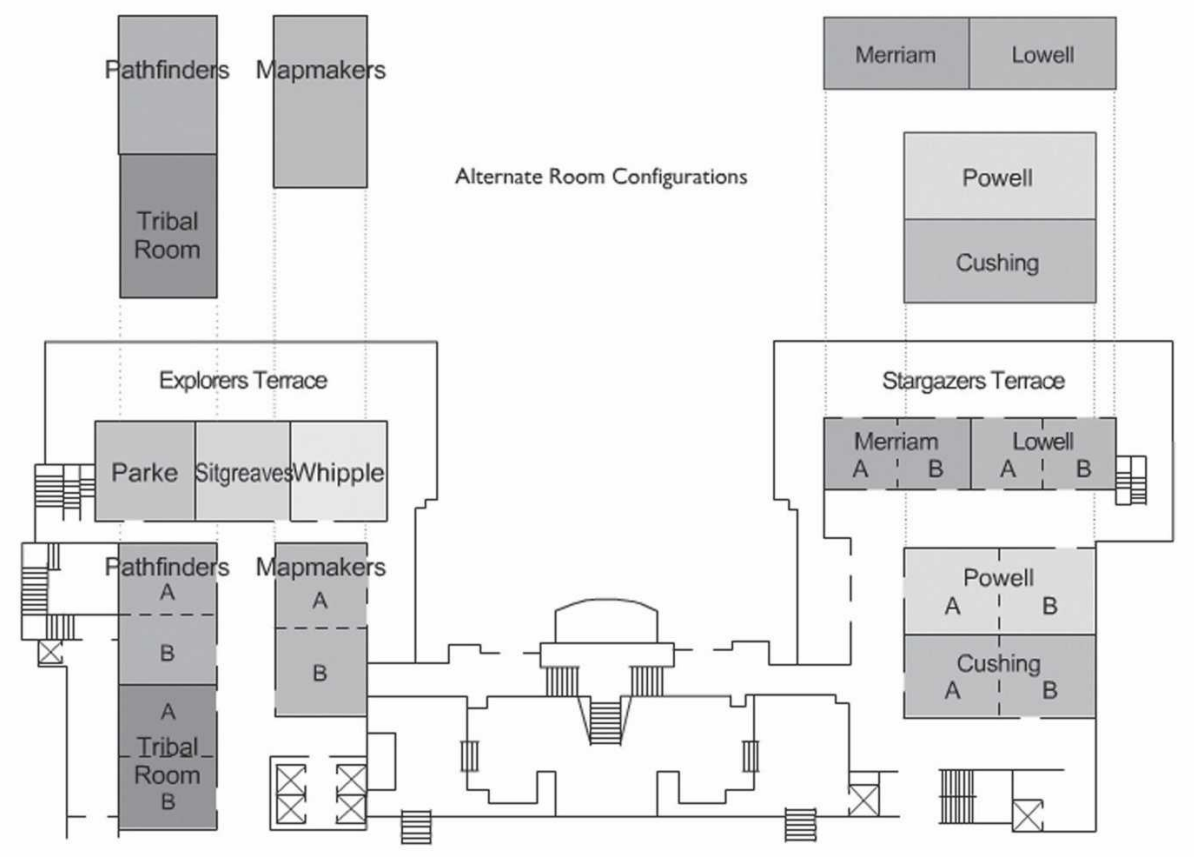




\section{Grand Ballroom Lobby Level}

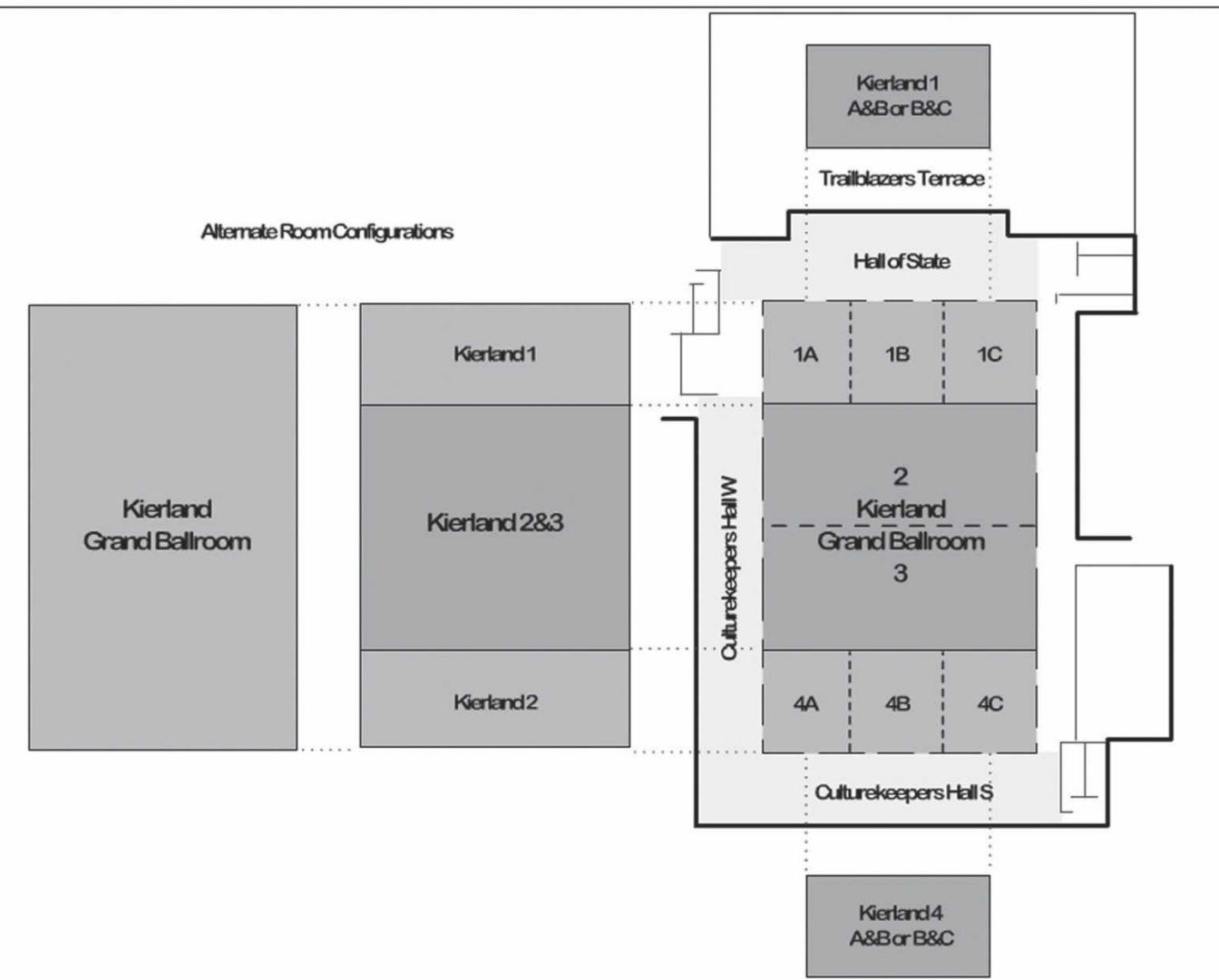

\section{Herberger Ballroom, Lower Level}

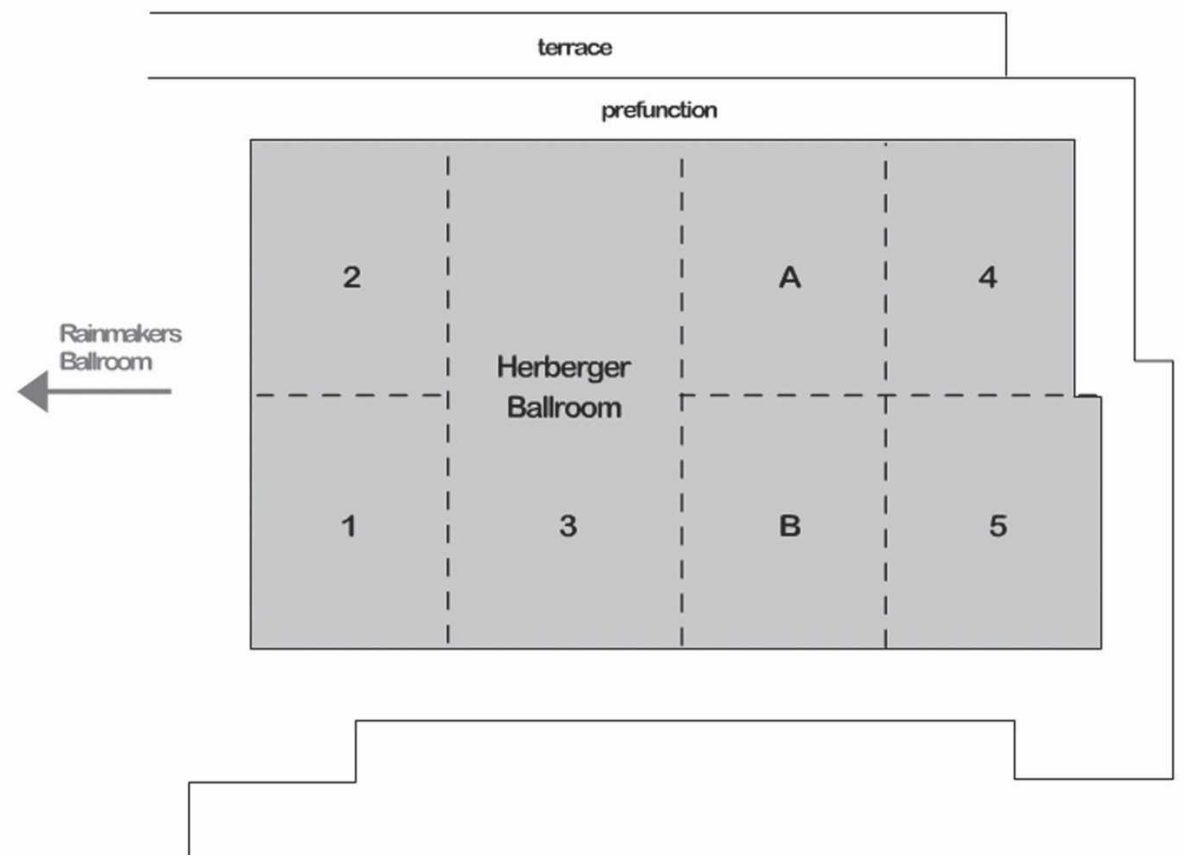


Close-up Exposition Hall (subject to change)

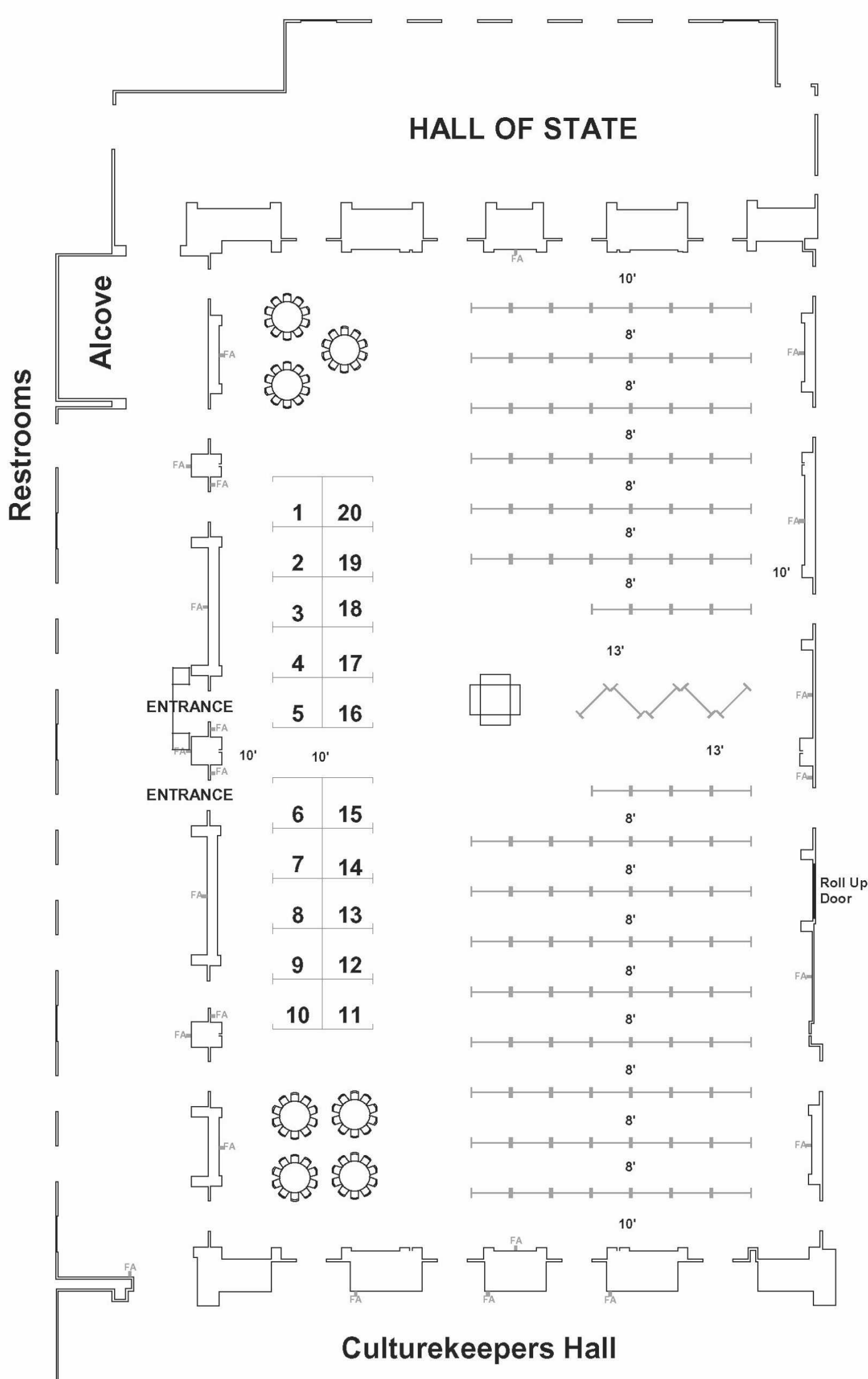




\title{
Guide to Exhibitors \\ ASHS-2007 • 16-19 July 2007
}

\author{
Westin Kierland Resort and Spa
}

The exhibits are located in the Kierland Grand Ballroom, convenient to the technical sessions and all other conference events. Exhibit Hall hours are:

Monday, 16 July 12:00-6:00 pm

Tuesday, 17 July 9:00 am-6:00 pm
Wednesday, 18 July 9:00 am-5:30 pm

Thursday, 19 July 9:00 am-2:00 pm

\section{Bio Chambers Incorporated}

Company Representative: Robert Pauls

477 Jarvis Avenue

Winnipeg, Manitoba R2W 3A8, Canada

Phone: 204-589-8900; Fax: 204-582-1024

E-mail: rob@biochambers.com

CID, Inc.

Booth \#4

Company Representative: Ying Yan

4845 NW Camas Meadows Dr.

Camas, WA 98607

Phone: 360-833-8835; Fax: 360-833-1914

E-mail: sales@cidinc.com

CID, Inc., designs and manufactures high technology research instruments. We conduct business around the world through a group of over 30 representatives and an international sales department that handles communications and relations with our representatives and clients to ensure that they are well provided for. We will demonstrate our Photosynthesis Systems, Leaf Area Meters, Plant Canopy Imager, Computer Image Analysis System, Soil Profile and Root Monitoring System, and Spectrometers.

\section{CRC Press}

\section{Booth \#13}

\section{Taylor and Francis Group, L.L.C}

Company Representative: John Sulzycki

6000 Broken Sound Parkway N.W., Suite 300

Boca Raton, FL 33487

Phone: 561-998-2507; Fax: 561-998-2559

E-mail: nancy.logal@taylorandfrancis.com
Web: www.crcpress.com

CRC Press-Taylor \& Francis Group, L.L.C., is a global publisher of scientific references and texts. Visit our booth and browse our books including our new Plant Propagation textbook. 15-25\% convention discount.

\section{CONVIRON}

Booth \#16

Company Representative: Laura Frederick 590 Berry Street Winnipeg, Manitoba R3H OR9 Canada Phone: 204-786-6451; Fax: 204-786-4433 E-mail: laura@conviron.com

CONVIRON manufactures equipment for research institutions and companies where the precise and simultaneous control of temperature, humidity, light, and other variable environmental conditions is essential to their success CONVIRON has expanded into the broader life science market, research greenhouses, and other applications where precise environmental control is essential.

Decagon Devices, Inc.

Booth \#15

Company Representative:

Tricia Oak

2365 NE Hopkins Court

Pullman, WA 99163

Phone: 509-332-2756

Fax: 509-332-5158

Decagon Devices - a leading manufacturer of plant, soil, and water instrumentation. Our instruments assist horticulturists in taking measurements such 
as stomotal conductance, EC, volumetric water content, water potential, and PAR/LAI. The booth will feature the all-new leaf Porometer, the easiest and most effective way to measure stomotal conductance, along with the WP4, AccuPAR LP-80, and $\mathrm{ECh}_{2} \mathrm{O}$ sensors.

\section{Environmental Growth Chambers Booth \#14}

510 East Washington Street

Chagrin Falls, OH 44022-4448

Phone: 800-321-6854

Fax: 440-247-8710

E-mail: sgriggs@egc.com

Web: www.egc.com

Environmental Growth Chambers (EGC) has over 50 years experience in the design and manufacture of controlled environment chambers. EGC has the largest selection of plant growth chambers for agriculture research of any company worldwide. We also produce tissue culture chambers, walk-in controlled environment rooms, lighted and refrigerated incubators, day-lit chambers, root zone cabinets, microprocessor, and central computer systems for control and monitoring. Please stop by to discuss your upcoming projects.

\section{LI-COR Biosciences}

Booth \#7

Company Representative: Chris Mantzios

4421 Superior Street

Lincoln, NE 68504

Phone: 402-467-3576; Fax: 402-467-0831

E-mail: envsales@licor.com

Web: www.licor.com

Visit LI-COR's booth to see the latest instrumentation for environmental research, including portable photosynthesis and fluorescence systems, dataloggers, leaf area meters, light meters, radiation sensors, and gas analyzers, including the LI-7000 precision $\mathrm{CO}_{2} / \mathrm{H}_{2} \mathrm{O}$ analyzer, and the new $\mathrm{LI}-840$ $\mathrm{CO}_{2} / \mathrm{H}_{2} \mathrm{O}$ analyzer.

\section{Longwood Graduate Program}

Booth \#6

Company Representative: Robert E. Lyons

University of Delaware

Newark, DE 19716

Phone: 302-831-2517

E-mail: rlyons@udel.edu

The Longwood Graduate Program prepares students to be leaders in public horticulture through University of Delaware coursework, thesis research, and close cooperation with Longwood Gardens. Students may also enrich their education with unique experiences involving the numerous public horticulture institutions within the Brandywine Valley of southeastern Pennsylvania and Delaware.

\section{Michigan State University-Horticulture Department Booth \#2}

Representatives: Yonathan Fridman,

Dario Stefanelli

Michigan State University-

Horticulture Department

A288 Plant \& Soil Science Building

East Lansing, MI 44824

Phone: 517-355-5191; fax: 517-355-0249

E-mail: stefanel@msu.edu

YODA ROOT ANALYZER ('Michigan State University) is software for automatic measurement of roots in any digital image format. With minimal user interaction, it accurately detects and analyzes roots as narrow as a pixel. Output lists root number, length, diameter, area, volume, branch number, and angle (92\% angle accuracy).

\section{Percival Scientific}

Booth \#11

Company Representative: Patrea Hill

505 Research Drive

Perry IA 50220

Phone: 800-695-2743

Fax: 515-465-9464

E-mail: sales@percival-scientific.com 


\section{Pearson Education}

One Lake Street

Upper Saddle River, NJ

Phone: 201-236-5894

Fax: 201-236-5888

\section{PhytoTechnology Laboratories $^{\mathrm{Tm}}$}

Booth \#3

Company Representative: Kenneth Torres 14335 West 97th Terrace

Lenexa, KS 66215

Phone: 913-341-5353; Fax: 931-544-0553

E-mail: ken@phytotechlab.com

PhytoTechnology Laboratories ${ }^{\mathrm{TM}}$ is a leading manufacturer and distributor of chemicals, media, and equipment and supplies for the plant tissue culture and plant science markets. Located in a suburb of Kansas City, most products are shipped within 24 hours and reach our customers in 2 business days.
Spectrum Technologies, Inc.

Booth \#12

Company Representative: Mike Thurow

12360 S. Industrial Drive East

Plainfield, IL 60585

Phone: 815-436-4440; Fax: 815-436-4660

E-mail: rebecca@agmeters.com

Spectrum Technologies, Inc., offers affordable devices to measure nutrient levels, soil qualities, light, weather, and other factors affecting plant growth. Our WatchDog ${ }^{\circledR}$ weather stations and data loggers make it easy to record weather events and conditions. Over 15,000 customers count on Spectrum's easy-to-use, dependable technology for their growing needs

\section{REAL-TIME WEATHER INFORMATION DIRECTLY FROM YOUR RESEARCH PLOTS}

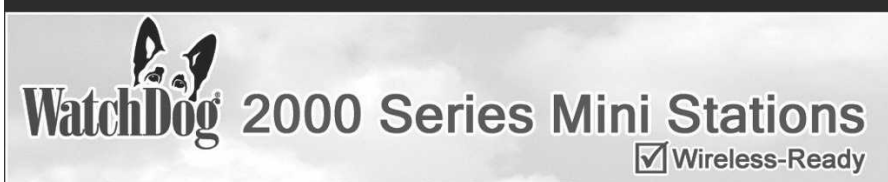

Professional grade solutions for:

- Plant growth monitoring

- Early pest/disease forecasting

- Efficient irrigation management

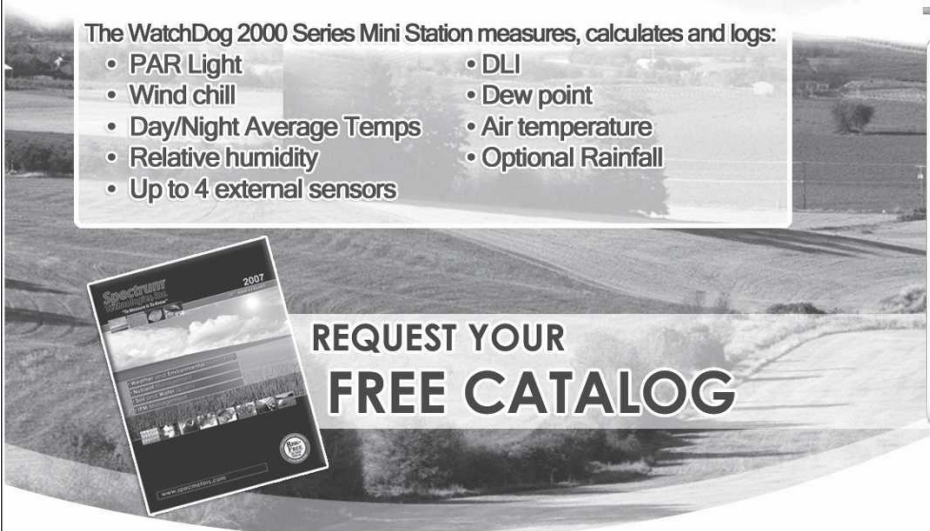

\section{Spectrum} Technologies, Inc.

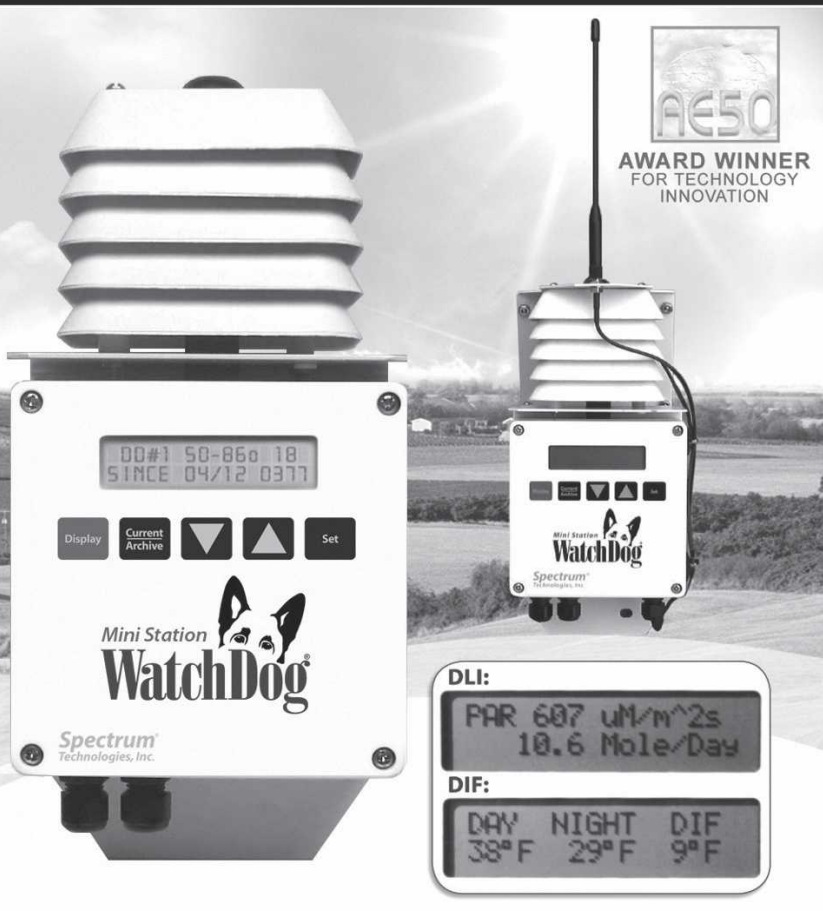

Visit us online at www.specmeters.com 


\section{The American Society for Horticultural Science would like to thank the following for their efforts in making this a successful conference:}

\section{Local Arrangements Committee}

Dennis Ray

Chieri Kubota

Mark Bierner

Also, special thanks go to

the following for making

this year's horticultural

tours a success:

Gene Giacomelli

Patricia Rorabaugh

Steve Carter

Kim Stone

EuroFresh Farms

Boyce Thompson Arboretum

Annual Conference Technical

Program Committee

Dennis T. Ray, Co-Chair

Carl E. Sams, Co-Chair

Erik Runkle

Rebecca Darnell

Jeff Anderson

Kirk Pomper

Julia Kornegay

Kathryn Orvis

Bala Rathinasabapathi

Fellows Screening Committee

Dewayne Ingram, Chair

Richard Fery

Fred T. Davies

Richard Zimmerman
Mike Chaplin

Donald Maynard

Helen Harrison

Mary L. Albrecht

George Hochmuth

William R. Woodson

Scholarship Awards

Committee

Janet Cole, Chair

Thomas Marler

Sorkel Kadir

Sanjun Gu

Holly Scoggins

Ryan Stewart

Bruno Moser

Paul Smeal

Collegiate Activities Committee

Richard Harkess, Chair

Terry Ferriss

Murali Panen

Bob Mirabelo

Tracy Dougher

Gary Bachman

Brian Trader

Rob Smith

Monica Robinson

Graduate Student Activities Committee

Barbara Liedl, Chair

Sudeep Vyapari

Robert R. Tripepi

Mark Ritenour
Mengmeng Gu

Chrislyn Particka

Selection Committees for Career Awards

Extension Educator Award

Essie Fallahi, Chair

Jim Ferguson

Rhoda Burrows

Mosbah Kushad

Kerrie Badertscher

International Horticulturist

Award

Tom Davenport, Chair

Guochen Yang

Essie Fallahi

Michael Raviv

Graduate Educator Award

Steven Sargent, Chair

Carolyn DeMoranville

Susan Brown

Paul Bosland

Carl Sams

Researcher Award

Greg Reighard, Chair

Brian Kahn

Joe Scheerens

Edward Hellman

Jack Staub

Alan Lakso

Robert Prange

Industry Scientist Award

Harold Fonda, Chair

Craig Campbell

James W. Rushing

Gary Hennen 


\section{The American Society for Horticultural Science would like to thank the following for}

their efforts in making this a successful conference:

$$
\begin{aligned}
& \text { James Watson } \\
& \text { Roger Bowman } \\
& \text { Nancy Roe }
\end{aligned}
$$

Undergraduate Educator

Award

Rolston St. Hilaire, Chair

Emily Hoover

Buddy Tignor

Todd West

David Himelrick

Robert Lyons

\section{Screening Committees for \\ Publication Awards}

Cross-Commodity Publication Award

Matthew Fidelibus, Chair

Bruce Schaffer

Caula Beyl

Gary Watson
Terry Howe

James Syvertsen

William Miller

Education Publication Award

Rick Durham, Chair

David Creech

George Fernandez

Terry Ferris

Janet Cole

David Reed

Ornamentals Publication

Award

Bob Stamps, Chair

Sherry Kitto

Sue Wiegrefe

John Dole
Frank Blazich

Theo J. Blom

Max Cheng

Extension Publication Award

Michael Parker, Chair

Scott Johnson

Jim Hruskoci

Paul Domoto

Brian Smith

Al Cooke

Franz Niederholzer

Vegetable Publication Award

Christine Coker, Chair

Wade Berry

Delbert Hemphill

Harlene Hatterman-Valenti

Mark Bennett

Mosbah Kushad

John Scott

\section{ASHS also thanks}

\section{Seminis Vegetable Seeds}

for sponsoring this year's William A. "Tex" Frazier Lecture

\section{and}

\section{Valent BioSciences Corporation}

for sponsoring this year's Extension \& Industry Dinner 


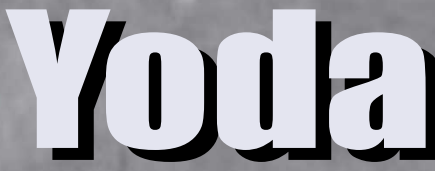

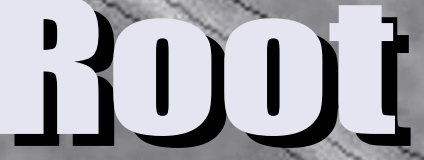 \\ (1)

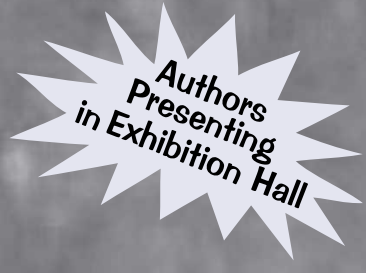

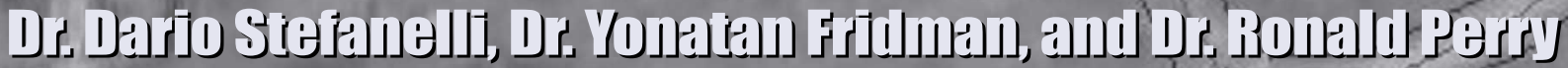

You design your own experimental paranseters... jeasurements are no longer restrictive.

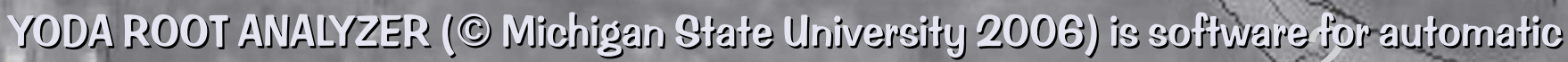

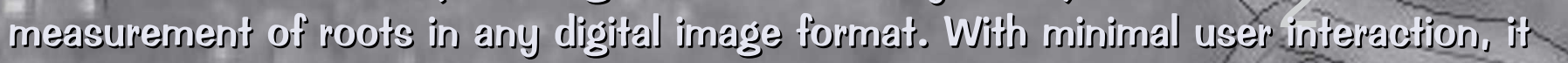
accurately defects and analyzes joots as najrow as a pixel. Oujpuj lists root nussber lengifh, oljameters, ajrea, volusse, branch number and angle.

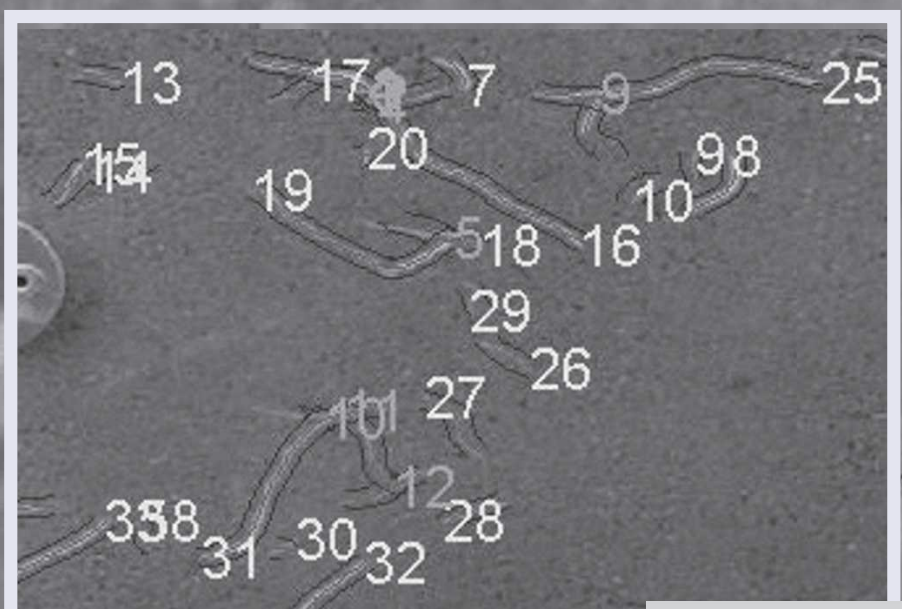

$\square \square \mathbf{X}$

24

For Ordering/Pricing Information

Instructional Media Center Michigan State University orders.oip.msu.edu or stefanel@msu.edu (product release date: 7/12/07)

\begin{tabular}{|c|c|c|c|}
\hline Radius & \multirow{2}{*}{$\stackrel{2}{2} 50$} & Class Divisions & \multirow{2}{*}{$\begin{array}{l}\text { Original Image Dimensions } \\
\begin{array}{|l}\square \\
\square\end{array} \text { y } \mathrm{mm} \quad \nabla\end{array}$} \\
\hline 504 & & 0.2 Diameter $(\mathrm{mm})$ & \\
\hline Step Size & 1 & 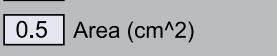 & $\square$ Invert Input Image \\
\hline $0.01 \square$ & D 10 & 0.05 Volume $\left(\mathrm{cm}^{\wedge} 3\right)$ & Dots Per Inch (DPI) \\
\hline Pre-Blur & 0 & \# of Branches & $\begin{array}{l}\text { Write To... } \\
\text { Wracking }\end{array}$ \\
\hline$0 \longdiv { 1 }$ & 10 & 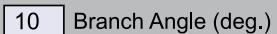 & $\mathrm{xls} \mathbf{\nabla}$ \\
\hline & & & Close \\
\hline
\end{tabular}

